This item was submitted to Loughborough's Research Repository by the author.

Items in Figshare are protected by copyright, with all rights reserved, unless otherwise indicated.

\title{
Gait analysis for designing a new assistive knee brace
}

PLEASE CITE THE PUBLISHED VERSION

http://dx.doi.org/10.1109/ROBIO.2011.6181583

\section{PUBLISHER}

(c) IEEE

\section{VERSION}

AM (Accepted Manuscript)

\section{PUBLISHER STATEMENT}

This work is made available according to the conditions of the Creative Commons Attribution-NonCommercialNoDerivatives 4.0 International (CC BY-NC-ND 4.0) licence. Full details of this licence are available at: https://creativecommons.org/licenses/by-nc-nd/4.0/

\section{LICENCE}

CC BY-NC-ND 4.0

\section{REPOSITORY RECORD}

Guo, Hongtao, Aaron See-Long Hung, Wei-Hsin Liao, Daniel Tik-Pui Fong, and Kai-Ming Chan. 2019. "Gait Analysis for Designing a New Assistive Knee Brace". figshare. https://hdl.handle.net/2134/21281. 


\title{
Gait Analysis for Designing a New Assistive Knee Brace
}

\author{
Hongtao Guo, Aaron See-Long Hung, Wei-Hsin Liao, Daniel Tik-Pui Fong, and Kai-Ming Chan
}

\begin{abstract}
Assistive knee brace is a species of wearable lower extremity exoskeletons. In this research, an assistive knee brace was developed by integrating a multifunctional actuator with a custom-made knee-ankle-foot orthosis. In the study, the location of the actuator is moved up to the lateral side of the hip, instead of knee joint. Waist belt and shoulder belt are appended on the knee brace. This paper aimed to improve the design of the assistive knee braces through gait analysis. By walking with the knee braces, the spatial and temporal gait parameters, joint kinematics and joint kinetics parameters were evaluated, and the changes from normal walking were compared as well. The experimental results showed that walking with the developed knee brace provided minimal hindrance to the wearer.
\end{abstract}

\section{INTRODUCTION}

A SISITIVE knee brace is a species of wearable lower extremity exoskeletons. Such assistive equipment can enhance people's strength and provide desired locomotion and have advantages over wheelchairs. It is possible to use knee braces to assist elderly or disabled people on improving their mobility in order to solve many daily life problems, such as going up and down stairs and crossing over obstacles. With an aging world population, devices that help elderly with mobility problems are in great need. By using assistive knee braces, patients may avoid being bedridden and will be able to maintain their physical activities and enjoy an active lifestyle.

Studies have been conducted in the development of exoskeletons, such as the RoboKnee developed by Pratt et al [1], the Hybrid Assistive Limb developed by Sankai et al [2], BLEEX by Kazerooni et al [3], MIT Exoskeleton by Herr et al [4] and the Wearable Walking Helper by Kosuge et al [5]. Companies like HONDA, Raytheon and Berkeley Bionics have also launched their products on exoskeletons or assistive knee braces [6-8]. Some researchers adopted active actuators

Hongtao Guo is with the Department of Mechanical and Automation Engineering, Faculty of Engineering, The Chinese University of Hong Kong, Shatin, N. T., Hong Kong (e-mail: htguo@mae.cuhk.edu.hk).

Aaron See-Long Hung is with the Department of Orthopaedics and Traumatology, Prince of Wales Hospital, Faculty of Medicine, The Chinese University of Hong Kong, Shatin, N. T., Hong Kong (e-mail: ahung @ort.cuhk.edu.hk).

Wei-Hsin Liao is with the Department of Mechanical and Automation Engineering, Faculty of Engineering, The Chinese University of Hong Kong, Shatin, N. T., Hong Kong (phone: +852 2609-8341; fax: +852 2603-6002; e-mail:whliao@ cuhk.edu.hk).

Daniel Tik-Pui Fong is with the Department of Orthopaedics and Traumatology, Prince of Wales Hospital, Faculty of Medicine, The Chinese University of Hong Kong, Shatin, N. T., Hong Kong (e-mail: dfong@ort.cuhk.edu.hk).

Kai-Ming Chan is with the Department of Orthopaedics and Traumatology, Prince of Wales Hospital, Faculty of Medicine, The Chinese University of Hong Kong, Shatin, N. T., Hong Kong (e-mail: kaimingchan@cuhk.edu.hk). such as electric motor, hydraulic and pneumatic cylinders as actuation device. Such actuators usually consume large power especially in brake function. However, power consumption in the actuation devices is an important factor in extending the working time of batteries after fully charged. According to Clinical Gait Analysis (CGA), it can be found that the knee joint is usually dissipating power during walking [9]. Hence, the knee joint dynamics could be matched by a controlled energy dissipative device, such as semi-active actuators. Several research groups have developed prosthetic knees or knee braces using MR fluids based devices [10-12]. However, in some situations such as climbing upstairs, the MR actuators will not be able to provide active torque to assist the wearer.

To combine the advantages of electric motors and MR fluids, Chen and Liao developed an assistive knee brace by integrating an MR actuator with electric motor [13]. With adaptive control, the actuation system worked well and could provide desired torque with better safety and energy efficiency. However, the actuator seemed a bit bulky to be used on human body. A more compact actuator is desired for assistive knee braces. With this motivation, a multifunctional actuator integrating motor, clutch and brake functions into a single device was designed. Guo and Liao developed the multifunctional actuators with inner armatures and input/output plates, respectively [14-15]. The actuator has multiple functions as motor, clutch and brake, and has the advantages of less power consumption, improved safety and better controllability. In this research, the assistive knee braces used this multifunctional actuator in the actuation system.

However, the assistive knee braces using the multifunctional actuator can only provide assistive torque in single degree of freedom (DOF). Since the human knee has three planes of motion (flexion/extension, valgus/varus, internal/external rotation), it makes the integration between the assistive knee brace and the wearer very challenging. Furthermore, the location of the actuator is another consideration for developing knee braces that provide smooth locomotion.

A prototype of the assistive knee brace adopted the multifunctional actuator, which was located at the lateral side of the knee joint was fabricated and tested [16]. Experiments were carried out to evaluate the kinematic and kinetic changes of walking with the assistive knee brace. The results showed that except for the knee flexion angle and the knee external and internal rotation angles, there were little changes in the spatial and temporal gait parameters, the joint kinematics and kinetics for walking with the knee brace when compared with 


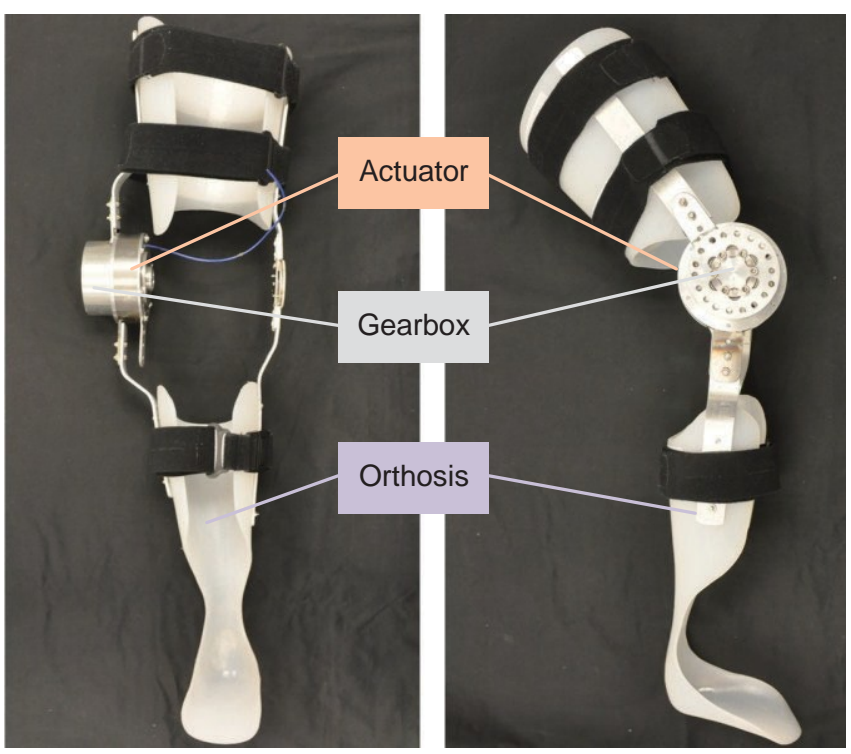

Fig. 1. The first prototype of the assistive knee brace.
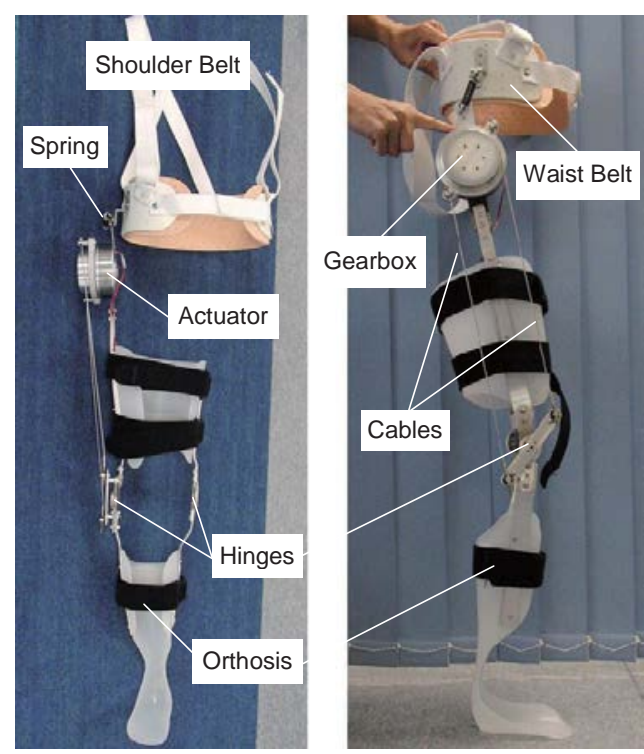

Fig. 2. The second prototype of the assistive knee brace. normal walking. The changes in the knee flexion and rotation angles were caused by an added weight to the knee joint which increased the inertia of the swing leg.

In this paper, the second prototype was fabricated and tested. The location of the actuator was moved up to the lateral side of the hip joint instead of the knee joint. Experiments were conducted to evaluate the spatial and temporal gait parameters, joint kinematics and joint kinetics parameters by walking with the knee braces and compared with normal walking. This evaluation will be used to identify possible areas for further improvement and better locations for the multifunctional actuator during the development of the assistive knee braces.

\section{II.EXPERIMENTS}

\section{A. Subject}

One healthy male (weight $60.0 \mathrm{~kg}$, height $175.0 \mathrm{~cm}$ ) participated in this experiment. The subject reported no previous lower limb injuries that might affect his gait.

\section{B. Assistive Knee Braces with Multifunctional Actuator}

Two prototypes of the assistive knee braces were fabricated as shown in Fig. 1 and Fig. 2. Each prototype

TABLE I

SPECIFICATIONS OF THE SECOND PROTOTYPE

\begin{tabular}{ll}
\hline \hline Materials & \\
Braces and waist belt & Polyethylene \\
Shafts and hinge & Aluminum \\
MR fluids & MRF-132DG \\
Cables & Steel \\
Weight & $2.74 \mathrm{~kg}$ \\
Height & $94.5 \mathrm{~cm}$ \\
Maximum width at hip joint & $62 \mathrm{~cm}$ \\
Thigh segment diameter & $58.6 \mathrm{~cm}$ \\
Calf segment diameter & $40.0 \mathrm{~cm}$ \\
Foot segment length & $14.8 \mathrm{~cm}$ \\
\hline \hline
\end{tabular}

consists of a custom-made knee-ankle-foot orthosis (KAFO), a multifunctional actuator, and a hinge joint at the medial side of the knee. In the first prototype, the actuator is located at the lateral side of the knee joint, while in the second prototype it is at the lateral side of the hip joint. The specifications of the second prototype that is adopted in this study are given in Table I.

In the second prototype, the multifunctional actuator is installed into the KAFO and its joint axis is aligned to the joint axis of the subject's hip joint. A hinge joint with bearing is installed to the KAFO at the knee joint position. One metal bar and two steel cables along with the cover of the multifunctional actuator form the planar four-bar linkages. As the four-bar linkages are a kind of parallelogram linkages, the output torque from the actuator is therefore transmitted to the hinge at the lateral of the knee joint. Another hinge joint is also installed to the medial side of the KAFO to increase the stability and performance of the assistive knee brace. To support the weight of the knee brace and the actuator, a waist belt and shoulder belt are added. A spring is adopted to connect the waist belt and knee braces. The subject with the assistive knee brace is shown in Fig. 3.

The multifunctional actuator used in this research is a novel actuator to integrate the advantages of electric motor and MR fluids while decreasing the dimension. By applying currents on different coils, the actuator could function as motor, clutch and brake. To investigate the interaction between the wearer and the knee braces, the multifunctional actuator is in 'off' mode in the experiments. Hence, this research evaluated the kinematic and kinetic changes of walking with the second prototype of the assistive knee brace in the "off" mode.

\section{Motion Capture}

Three-dimensional motions of the subject were measured 


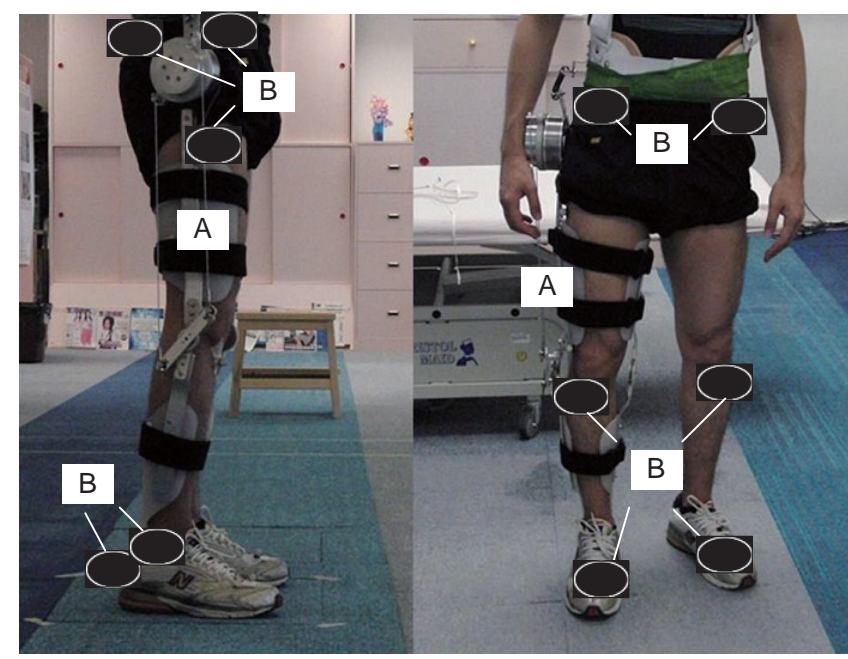

Fig. 3. Integration of knee brace to the wearer and placement of reflective markers (A - Knee brace; B - Reflective markers).

using an optical motion analysis system with 8 cameras (VICON 624, Vicon Motion Systems Ltd, Oxford, United Kingdom) at $120 \mathrm{~Hz}$ as shown in Fig 4. The motion capture system was synchronized with a force platform (model OR6-7, AMTI, Watertown, Massachusetts) at a capturing frequency of $120 \mathrm{~Hz}$. Sixteen skin reflective markers (9 mm in diameter) were attached at anatomical landmarks of the lower body according to the Vicon Plug-in Gait model, which included the anterior superior iliac spine, the posterior superior iliac spine, the greater trochanter, the lateral femoral condyle, the tibial tuberosity, the lateral malleolus, the heel and the fifth metatarsal on both right and left sides as shown in Fig. 3. All the markers were attached by the same tester.

\section{Protocol}

The subject was instructed to walk along a straight eight meter path without the assistive knee brace and while wearing the knee brace. In order to perform inverse dynamics to calculate the lower limb joint kinetics (i.e. joint moments, joint force), only trials in which the right leg of the subject completely landed on the force platform were used. To ensure that the subject walked naturally, the location of the force platform on the floor was not disclosed to the subject. Instead, the subject was instructed to begin walking at different starting positions.

\section{E. Data Analysis}

\section{1) Spatial and Temporal Gait Parameters}

Three successful trials from normal walking and three other successful trials from walking with the knee brace were used. The spatial and temporal gait parameters were analyzed according to Vaughan et al (1992) [17]. In order to compare the two walking conditions, the data were time normalized to $100 \%$ of the gait cycle. Heel strike and toe off were determined with a $20 \mathrm{~N}$ threshold vertical force. Stride length was determined using the left and right toe markers between successful heel-strikes. Cadence was calculated by dividing

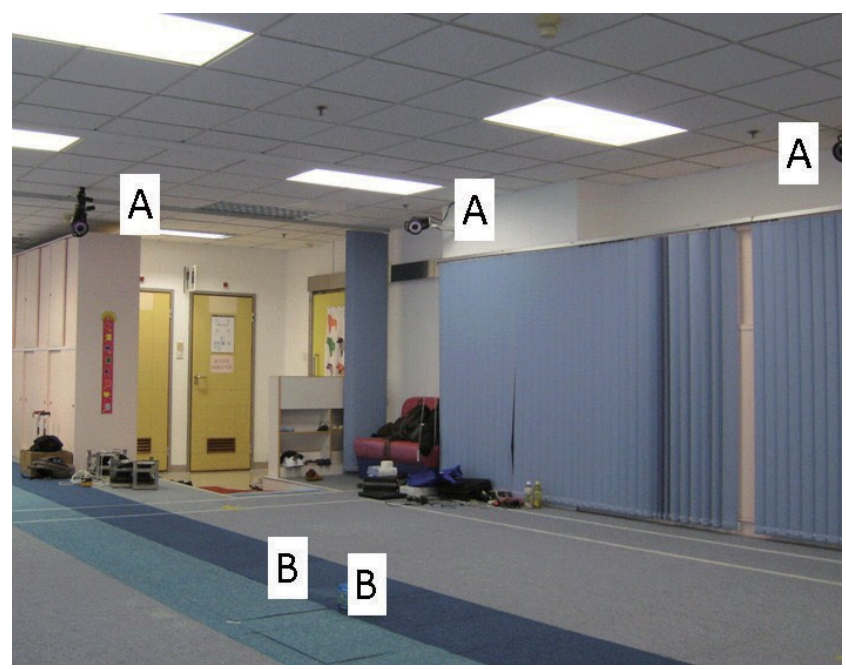

Fig. 4. Experimental setup (A - Infra-red cameras; B - Embedded force platforms)

the total steps taken by the total time. The average walking speeds in the two trials were calculated by dividing the total distance travelled by the time.

\section{2) Joint Kinematics and Kinetics}

Joint kinematics was analyzed for the entire gait cycle whereas joint kinetics was only analyzed for the stance phase. The data were analyzed using the Vicon Plug-in Gait model and were Butterworth filtered at a cut-off frequency of $10 \mathrm{~Hz}$. Average curves were formed for the vertical ground reaction force (vGRF) between normal walking and walking with the knee brace. Maximum vGRF and maximum joint ranges of motion (ROM) were also determined for comparison.

\section{F. Statistical Analysis}

The differences between maximum vGRF, joint ranges of motions and joint moment between normal walking and walking with the knee brace were analysed using paired t-tests. The level of significance was set at $\mathrm{p} \leq 0.05$.

\section{RESUlTS}

\section{A. Spatial and Temporal Gait Parameters}

As shown in Table II, the walking cycle for both conditions is 1.06 seconds and the average speed is 1.4 meters per second. No significant differences were found for the spatial and temporal gait parameters. It indicated that the subject's locomotion was not significantly affected by wearing the knee brace from these gait parameters.

TABLE II

SPATIAL-TEMPORAL GAIT PARAMETERS

\begin{tabular}{lll}
\hline & Normal walking & Walking with knee brace \\
\hline Cycle time $(\mathrm{s})$ & $1.06(0.006)$ & $1.06(0.01)$ \\
Cadence $(\mathrm{steps} / \mathrm{min})$ & $113.33(1.53)$ & $113.33(1.15)$ \\
Stride length $(\mathrm{m})$ & $0.76(0.02)$ & $0.75(0.015)$ \\
Average speed $(\mathrm{m} / \mathrm{s})$ & $1.40(0.02)$ & $1.39(0.04)$ \\
\hline
\end{tabular}


TABLE III

JOINT RANGES OF MOTION BETWEEN NORMAL WALKING AND WALKING WITH KNEE BRACE

\begin{tabular}{|c|c|c|c|c|c|}
\hline & Normal walking & SD & Walking with knee brace & SD & p value \\
\hline Hip flex/ext (deg) & 45.42 & 1.67 & 48.63 & 1.13 & $0.031^{*}$ \\
\hline Hip abd/add (deg) & 11.34 & 0.34 & 9.75 & 1.41 & 0.231 \\
\hline Hip int/ext rot (deg) & 11.77 & 1.62 & 15.82 & 1.46 & 0.051 \\
\hline Knee flex/ext (deg) & 65.04 & 2.44 & 56.8 & 1.88 & $0.028 *$ \\
\hline Knee val/var (deg) & 7.36 & 0.52 & 19.66 & 0.79 & $0.002 *$ \\
\hline Knee int/ext rot (deg) & 11.86 & 1.02 & 17.1 & 1.23 & $0.047 *$ \\
\hline Ankle plant/dorsi flex (deg) & 55.68 & 3.57 & 41.39 & 3.05 & $0.032 *$ \\
\hline Ankle inv/ev (deg) & 17.42 & 3.06 & 30.26 & 3.5 & $0.03^{*}$ \\
\hline Ankle int/ext rot (deg) & 9.35 & 2.27 & 8.22 & 1.26 & 0.504 \\
\hline
\end{tabular}

\section{B. Kinematic Parameters}

The overall patterns of the averaged joint kinematic curves for comparison are shown in Fig. 5. Similar movement patterns were found for the hip, knee and ankle joints. During walking, the knee brace restricted the sagittal ROM in the knee and ankle joints. Motions in sagittal plane are required during walking. Therefore, the knee brace affected normal walking gait. As shown in Table III, motions at the knee and ankle joints are affected by the knee brace. The subject had to compensate for the reduced motion. But, this time because the actuator is no longer at the knee, the subject compensated by adding extra motions at the knee joint (particularly at the frontal and transverse planes). The second prototype is different from the first one, where the subject compensated by rotating at the hip, but cannot claim that it is better than the first prototype. However, the differences were only 1 to 2 degrees.

\section{Kinetic Parameters}

Fig. 6 shows the vertical GRF differences between normal walking and walking with the knee braces. Quantitatively, the differences were found at the stages of heel-strike and toe-off. The greatest difference is about $20 \% \mathrm{BW}$. Table IV shows the mean differences and $\mathrm{p}$ value. It shows that there are some significant differences happed for the maximum vertical ground reaction force $(\mathrm{p}=0.005)$.

\section{DISCUSSION}

In our research, the second prototype of the assistive knee braces was designed and fabricated. The main difference between this prototype and the previous one is the location of the actuator. Different from the prototype adopted in [16] where the actuator was installed on part of knee joint, the
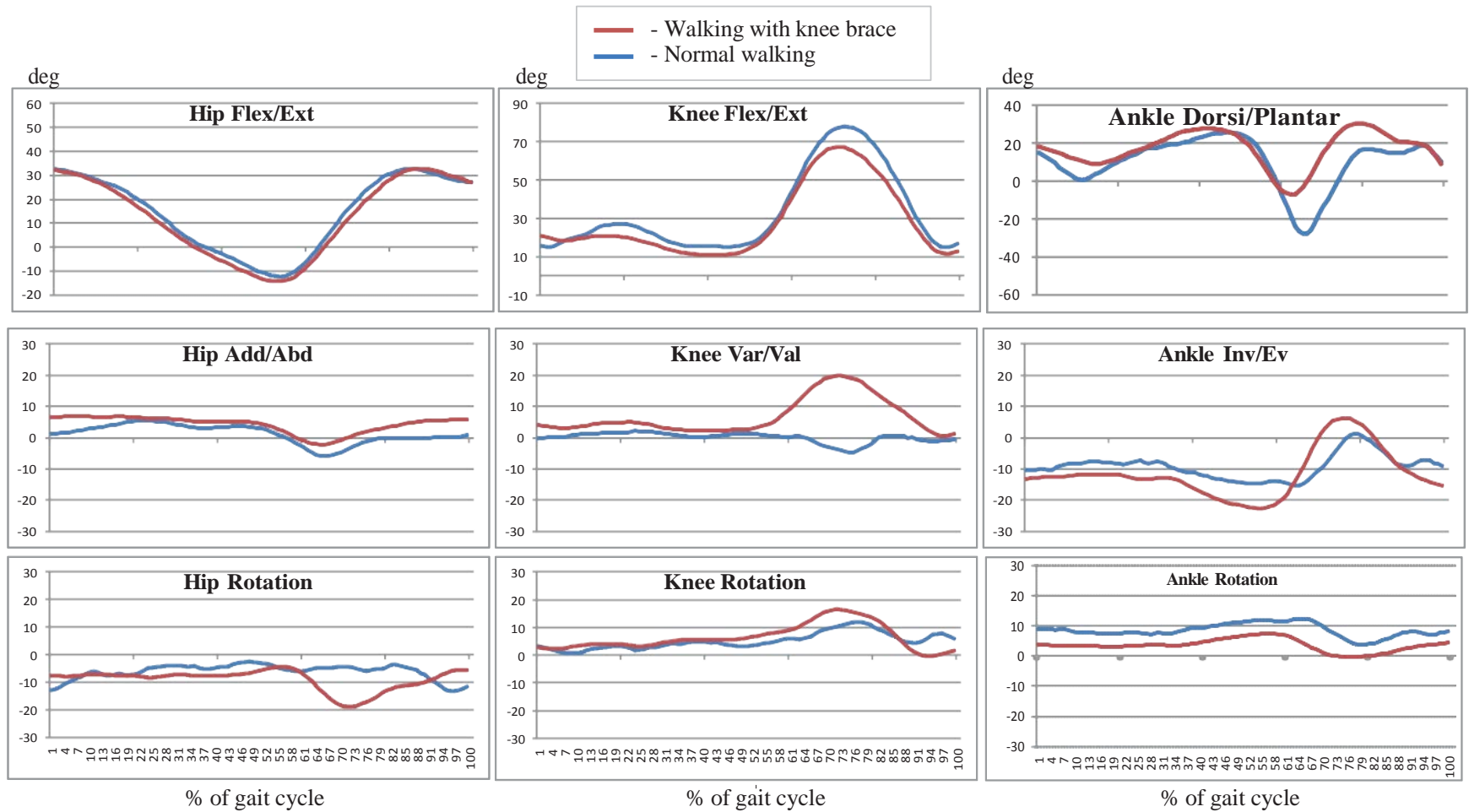

$\%$ of gait cycle

$$
\% \text { of gait cycle }
$$

$\%$ of gait cycle

Fig. 5. Joint kinematic profiles of normal walking and walking with the knee brace. 


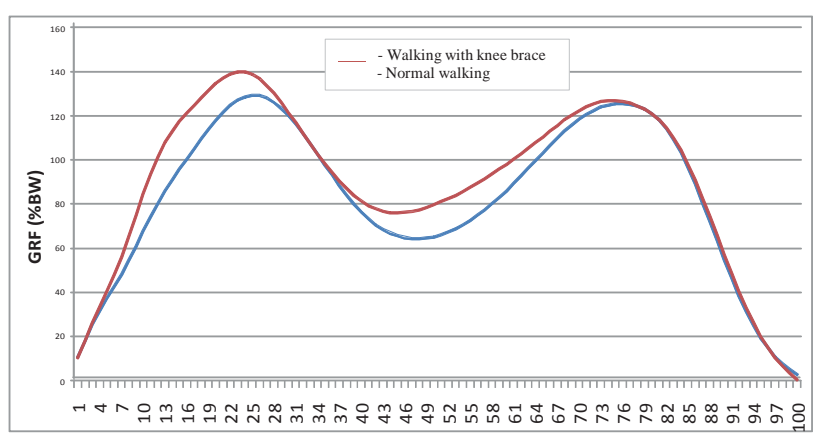

Fig. 6. Comparison of vertical GRF between two walking conditions.

actuator used in this experiment was installed on the part of hip. As the human knee joint has motions in three planes (flexion/extension, valgus/varus, internal/external rotation), this single DOF assistive torque should only be provided when motions at the other two planes are at a minimum. In order to identify the specific time in which the assistive torque should be provided, it is important to firstly identify changes in the gait pattern when walking with the knee brace. Any changes or hindrance caused by the knee brace should be identified before torque is applied to the knee joint.

Fig. 7 demonstrates the main gait cycle events between the two walking conditions. In general, our findings demonstrated little changes in the spatial and temporal gait parameters, the joint kinematics, and the joint kinetics for the wearer with the knee brace when compared with normal walking, except for the knee flexion angle and the knee external and internal rotation angles. There were significantly greater motions in the frontal planes for the knee and ankle, and greater motions for the transverse plane of the knee. These motions are most likely due to the subject's need to
TABLE IV

VERTICAL GRF DIFFERENCES BETWEEN NORMAL WALKING AND WALKING WITH KNEE BRACE

\begin{tabular}{lcll}
\hline \hline & Mean difference & SD & p value \\
\hline Vertical GRF max $(\% B W)$ & -10.16 & 1.29 & $0.005^{*}$ \\
\hline \hline * Significantly different from normal walking with p 0.05 &
\end{tabular}

maintain clearance. Therefore, motions at the knee and ankle joints are affected by the knee brace. The subject had to compensate for the reduced motion. But, it is different from the previous prototype, where the subject compensated by rotating at the hip.

It seems that the knee brace still restricted motion and affected normal walking gait. However, because the actuator is no longer at the knee joint, the subject adopted a different strategy to compensate for the restricted movement. The observed changes in the knee flexion and knee rotation angles could be attributed to the weight and friction caused by the multifunctional actuator and the steel cable. Different from the first prototype where the added load was placed to the knee joint so as to increase the inertia of the swing leg, in the second prototype waist belt and shoulder belt were applied to the knee brace so as to compensate the weight at knee joint. Therefore, in the stage of mid-swing, it can be found the vGRF in walking with the knee brace is the same as normal walking.

However, the weight of actuator still affected the whole system. The subject added extra motions particularly at the frontal and transverse planes in stages of heel-strike and toe-off, respectively. This was different from the first prototype, in which the subject externally rotated at the hip in order to achieve ground clearance. By placing the actuator at the hip, the restrictions of the range of motion at the knee

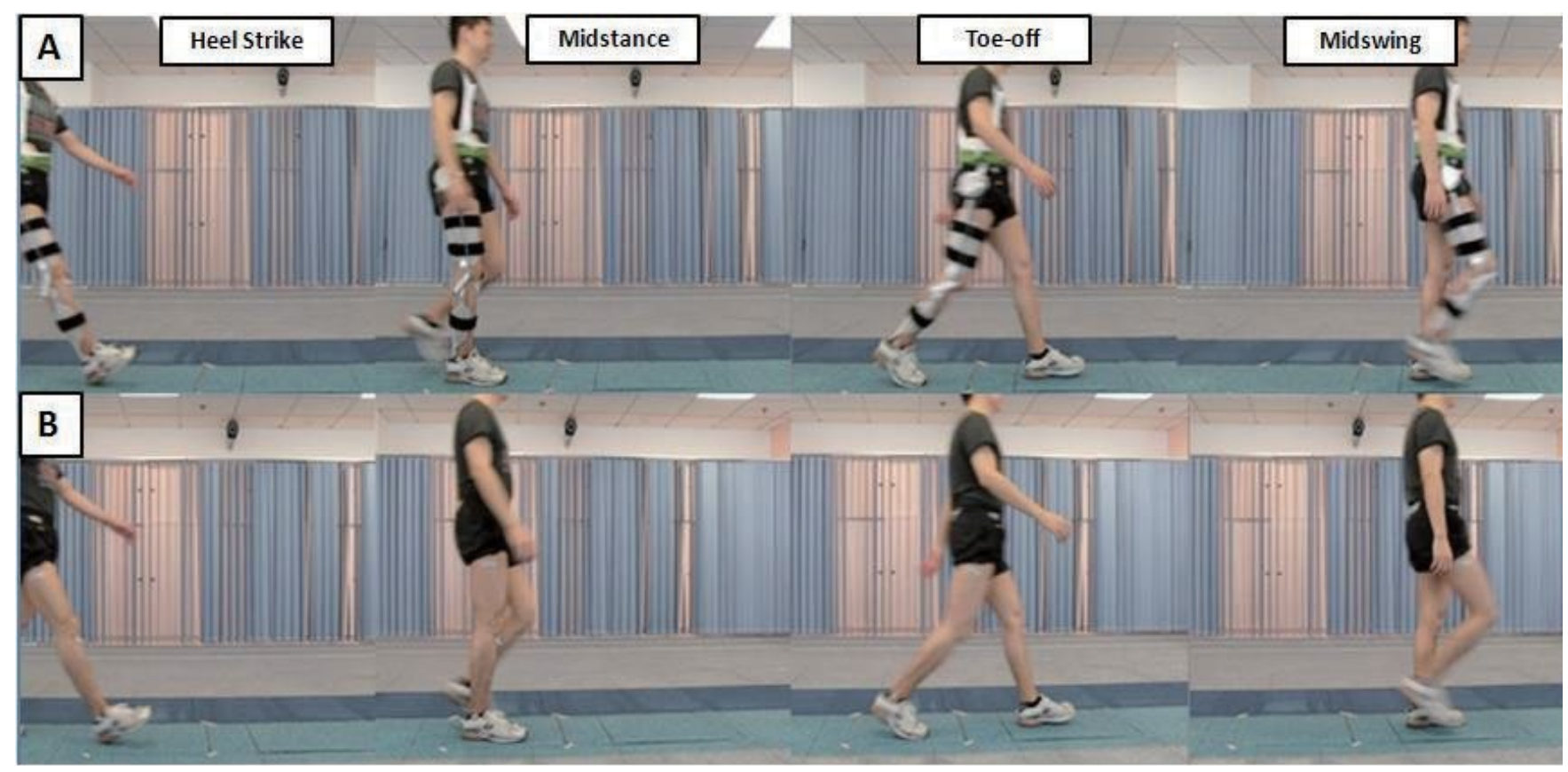

Fig. 7. Gait cycle events for two walking conditions (A - walking with knee brace; B - normal walking). 
were reduced. As a result, the subject was able to compensate his abnormal gait pattern at the knee joint, rather than at the more proximal hip joint. Therefore, the subject had to alter his gait patterns in both prototypes in order to account for the added friction at the actuator.

Since active torque could be provided by our assistive knee brace, some of these changes caused by the friction within the actuator could be attenuated.

Similar to the first prototype, a KAFO was designed according to the subject's anthropometric measurements. This allowed firm attachment to the subject's lower limb and greatly reduced the amount of shifting that occurred as the leg was in motion. Also, the KAFO along with the belts were able to partially support some of the weight of the multifunctional actuator. In the KAFO, the lower leg segment is attached with the foot segment. Thus when the subject was in stance phase, some of the weight of the actuator is transferred to the ground. However, with this design, the ankle range of motion was also compromised, as evident by the several degrees decreased in plantar flexion motion. Nonetheless, this decrease in ankle range of motion did not transfer to the ankle joint moment. This could be due to the mechanical structure of the orthosis, which produced an assistive plantar flexion moment as the orthosis restored to its original shape after it was bent.

Moreover, when the multifunctional actuator was integrated to the KAFO, it was aligned to the subject's hip joint center. During the design of the assistive knee brace, careful considerations and repeated testing were performed to ensure that motion of the actuator was confined in the sagittal plane. However, it was still not possible to replicate the complex human joint motion with only a single DOF joint.

\section{V.CONCLUSION}

This research experimentally investigated the interaction between the wearer and the developed knee brace during walking. Two prototypes were designed and fabricated. In this paper, the second prototype with actuator on hip was adopted. The assistive knee brace was developed by integrating the actuator with a custom-made knee-ankle-foot orthosis in order to minimize excessive shifting and to improve alignment to the knee joint. A comprehensive gait analysis was performed to evaluate changes in the walking gait caused by the developed knee brace in two walking conditions. From the experimental results, the second prototype did not show better performances than the first one in gait analysis. However, results indicated that the knee brace without actuation provided minimal hindrance to the user and assured that assistive torque can be applied to the knee joint while controlling the actuator.

\section{ACKNOWLEDGMENTS}

The work described in this paper was supported by a grant from the Innovation and Technology Commission of the Hong Kong Special Administrative Region, China (Project No. ITS/308/09).

\section{REFERENCES}

[1] J. Pratt, B. Krupp, C. Morse, and S. Collins, "The RoboKnee: an exoskeleton for enhancing strength and endurance during walking," in Proceedings of 2004 IEEE International Conference on Robotics and Automation, 2004. ICRA'04, vol. 3, 2004.

[2] Y. Sankai, "HAL: hybrid assistive limb based on cybernics," Robotics Research, vol. 66, pp. 25-34, 2011.

[3] H. Kazerooni and R. Steger, "The Berkeley lower extremity exoskeleton," Transactions of the ASME, Journal of Dynamic Systems, Measurements, and Control, vol. 128, pp. 14-25, 2006.

[4] C. J. Walsh, D. Paluska, K. Pasch, W. Grand, A. Valiente, H. Herr, "Development of a lightweight, underactuated exoskeleton for loadcarrying augmentation," in Proceedings 2006 IEEE International Conference on Robotics and Automation, pp.3485-3491, 2006.

[5] Y. Hirata, T. Iwano, and K. Kosuge, "Control of wearable walking helper on slope based on integration of acceleration and GRF information," in Proceedings of IEEE/RSJ International Conference on Intelligent Robots and Systems, pp. 3731-3736, 2008.

[6] HONDA, (7 Nov 2008) Honda unveils experimental walking assist device with bodyweight support system [Online]. Available: http://world.honda.com/news/2008/c081107Walking-Assist-Device/.

[7] Raytheon, Time Magazine Names the XOS 2 Exoskeleton "Most Awesomest" Invention of 2010 [Online]. Available: http://www.raytheon.com/newsroom/technology/rtn08_exoskeleton/.

[8] Berkeley Bionics, Berkeley Bionics Exoskeletons [Online]. Available: http://berkeleybionics.com/exoskeletons/.

[9] A. Zoss and H. Kazerooni, "Design of an electrically actuated lower extremity exoskeleton,” Advanced Robotics, vol. 20, no. 9, pp. 967-988, 2006.

[10] A. Wikenfeld and H. Herr, "Usear-adaptive control of a magnetorheological prosthetic knee," Industrial Robot: An International Journal, vol. 30, no. 1, p. 42-55, 2003.

[11] F. Ahmadkhanlou, J. Zite, and G. Washington, “A magnetorheological fluid-based controllable active knee brace," in Proceedings of SPIE, vol. 6527, 2007, p. 652700.

[12] K. H. Gudmundsson, F. Jonsdottir and F. Thorsteinsson, "A geometrical optimization of a magneto-rheological rotary brake in a prosthetic knee," Smart Materials and Structures, vol. 19, pp. 035023, 2010.

[13] J. Z. Chen and W. H. Liao, "Design, testing and control of a magnetorheological actuator for assistive knee braces," Smart Materials and Structures, vol. 19, 035029, 2010, doi:10.1088/09641726/19/3/035029.

[14] H. T. Guo and W. H. Liao, "Integrated design and analysis of smart actuators for hybrid assistive knee braces," Proceedings of SPIE Conference on Smart Structures and Materials: Active and Passive Smart Structures and Integrated Systems, vol. 7288, 2009, 72881U1-11, doi: 10.1117/12.815964.

[15] H. T. Guo and W. H. Liao, "Design and control of multifunctional magnetorheological actuators for assistive knee braces," in Proceedings of SPIE Conference on Smart Structures and Materials: Active and Passive Smart Structures and Integrated Systems, vol. 7643, 2010, 764310, doi: 10.1117/12.847530.

[16] A. S. L. Hung, H. T. Guo, W. H. Liao, D. T. P. Fong, K. M. Chan, "Experimental studies on kinematics and kinetics of walking with an assistive knee brace," in Proceedings of IEEE International Conference on Information and Automation (ICIA), pp. 45-50, 2011 doi: 10.1109/ICINFA.2011.5948961.

[17] C. L. Vaughan, B. L. Davis, and J. C. O’Connor, Dynamics of Human Gait, Human Kinetics Publishers Champaign, 1999. 